\title{
Responsabilidad social y política ambiental: complementariedades y retos
}

\author{
MANUEL PULGAR-VIDAL*
}

\begin{abstract}
SUMARIO:I.MÁS ALLÁDELSIMPLECUMPLIMIENTO DELALEY.-II.LASHERRAMIENTAS DEL DESARROLLOSOSTENIBLE.- III. UNA ACCIÓN VOLUNTARIA.- IV. ENTENDIENDO ELENTORNOY PROMOVIENDOLAPARTICIPACIÓN.-V.LARESPONSABILIDAD SOCIAL COMO PRÁCTICA INCLUSIVA.- VI. CONCLUSIONES.
\end{abstract}

Cuando uno reflexiona sobre la existencia o no de una política ambiental en un país como el Perú, puede plantear dos aproximaciones. La política existe porque ha sido expresamente aprobada mediante un decreto supremo ${ }^{1}$ y tiene un objetivo claro: mejorar la calidad de vida de las personas, al garantizar la existencia de ecosistemas saludables, viables y funcionales en el largo plazo; y el desarrollo sostenible del país, mediante la prevención, la protección y la recuperación del ambiente y de sus componentes, la conservación y el aprovechamiento sostenible de los recursos naturales, de una manera responsable, congruente con el respeto de los derechos fundamentales de la persona.

Otros podrían señalar que el Perú cuenta con ciertos elementos que permitirían plantear una política ambiental en construcción, porque esta no deriva de textos escritos y aprobados oficialmente, sino de un conjunto de elementos sin cuya articulación no hay propuesta política o legal que pueda hacerse cumplir.

De manera personal, a lo largo de los años he creído que los elementos básicos para pensar en la existencia de una política ambiental en el país son (a) regulaciones, bajo la forma de leyes, normas reglamentarias y otras normas complementarias, elaboradas sobre la base de información técnica y científica sólida, y en el pleno conocimiento de una realidad geográfica compleja y de una realidad social que nos reconoce como país pluri y multicultural; (b) institucionalidad con capacidad para gestionar las regulaciones, generar su cumplimiento y facilitar su entendimiento. En lo ambiental, ello demanda la organización de la institucionalidad en la lógica de un sistema funcional, que articule a los distintos órganos que ejercen determinadas funciones y que deben regirse por las directrices y los lineamientos de un ente rector; (c) mecanismos que aseguren la participación ciudadana en sus tres vías: acceso a la información, oportuna y a costo real, acceso al proceso y acceso a la justicia, que reclama reconocer el principio de patrocinio de intereses difusos, la existencia de

* Es abogado de la Pontificia Universidad Católica del Perú y profesor de los cursos de Derecho Ambiental, y Gestión y Manejo de los Recursos Naturales. Es director ejecutivo de la Sociedad Peruana de Derecho Ambiental.

1 La Política Nacional del Ambiente fue aprobada por el decreto supremo 012-2009-minAm del 22 de mayo de 2009. 
mecanismos procesales idóneos para atender estos intereses y entidades jurisdiccionales preparadas, capacitadas y especializadas en el tema; (d) mecanismos financieros para sustentar el costo de la implementación de la política ambiental, bajo reglas de transparencia y a través de mecanismos idóneos para el buen uso y el destino de los recursos; (e) formación, capacitación y difusión, orientados hacia la formación de la conciencia pública sobre las reglas ambientales y su cumplimiento; (f) incentivos orientados a premiar comportamientos ambientalmente sanos; y (g) voluntad política.

Los elementos no varían significativamente cuando nos referimos a responsabilidad social empresarial, definida como «la forma de gestión que se define por la relación ética y transparente de la empresa con todos los públicos con los cuales se relaciona y por el establecimiento de metas empresariales sostenibles con el desarrollo sostenible de la sociedad, al preservar recursos ambientales y culturales para generaciones futuras, y al respetar la diversidad y promover la reducción de las desigualdades sociales» ${ }^{2}$.

Es claro que, tan igual cuando nos referimos a la política ambiental, la responsabilidad social no es la que deriva de un documento aprobado en el ámbito de la corporación. Es más bien una filosofía, un modo de actuación, una actitud que conduce al reconocimiento por parte del entorno en el cual se desenvuelve la actividad empresarial.

Esta descripción inicial de los dos conceptos nos permite entender su complementariedad, pero a su vez nos permite entender las dificultades de su integración. Para ello cabe formular algunas preguntas a partir de cuya respuesta identificaremos los retos a los que nos enfrentamos en el mundo de hoy. iBasta cumplir la ley ambiental para ser socialmente responsable?, ies suficiente ser socialmente responsable para tener una conducta ambientalmente correcta?, iuna estrategia de responsabilidad social conduce al desarrollo sostenible?

\section{MÁS ALLÁ DEL SIMPLE CUMPLIMIENTO DE LA LEY}

Los abogados solemos asociar política ambiental a la legislación ambiental y a la institucionalidad creada para el ejercicio de funciones ambientales. Cuando pensamos en los avances de la política ambiental en el Perú, de inmediato recurrimos a la profusa legislación ambiental, que, en especial desde 1990, se ha promulgado en el país. Así reconocemos que la Constitución Política de 1993 reconoce el derecho a un ambiente sano como derecho fundamental de la persona ${ }^{3}$. Agregamos a ello el hecho de contar con una Ley General del Ambiente del año 2005, que

2 INSTITUTO ETHOS. Indicadores ETHOS de responsabilidad social empresarial. Instituto Ethos de Empresas e Responsabildade Social. Versión 2007.

3 Derecho que, a su vez, fue reconocido en la Constitución de 1979, aunque no expresamente como derecho fundamental de la persona. 
remplazó al emblemático Código del Medio Ambiente y los Recursos Naturales de 1990. Sabemos que contamos con una Ley Orgánica para el Aprovechamiento Sostenible de los Recursos Naturales, leyes sobre áreas naturales protegidas, diversidad biológica, evaluación del impacto ambiental, manejo de residuos sólidos, regulaciones sectoriales ambientales en minería, hidrocarburos, electricidad, industria manufacturera, entre muchas otras regulaciones, y obviamente reconocemos al Ministerio del Ambiente como la autoridad ambiental nacional.

Pero ni siquiera la existencia de esta normatividad o la creación de una institucionalidad ambiental a nivel de ministerio nos hacen pensar que tenemos una política ambiental nacional. Ni siquiera el cumplimiento de este marco legislativo nos llevaría a esa conclusión. ¿Por qué? Por varios motivos.

El primero es que, en materia ambiental, la legislación se ha enfocado básicamente en instrumentos, antes que en derechos. Así solemos preguntarnos si la actividad cuenta con un estudio de impacto ambiental, si está ejecutando el plan de manejo del Programa de Adecuación y Manejo Ambiental o si está cumpliendo con los límites máximos permisibles, pero poco nos preguntamos si está garantizado el derecho fundamental de la persona.

Eso diferencia al Derecho ambiental de la protección de los derechos humanos, e incluso de la defensa de los derechos del consumidor: el carácter legalista e instrumentalista del Derecho.

Orellana ${ }^{4}$ reconoce esta diferencia cuando introduce la complejidad en la relación entre el Derecho internacional de los derechos humanos y el Derecho ambiental, al señalar que:

[...] por ejemplo, el derecho internacional de los derechos humanos adopta un enfoque antropocéntrico, donde la realidad se aproxima principalmente desde el valor de lo humano. El derecho internacional ambiental en cambio oscila entre un enfoque antropocéntrico y un enfoque biocéntrico, donde la naturaleza adquiere valor en sí misma y no sólo en medida de su utilidad para la humanidad. Asimismo, los derechos humanos utilizan el lenguaje de «derechos», en cuanto valores básicos asociados a la dignidad humana, mientras que el derecho ambiental emplea un lenguaje de «estándares», a través del cual se determinan la calidad ambiental, límites a las emisiones contaminantes, etc. Estas diferencias en enfoque y lenguaje reflejan cierta distancia entre los dos regímenes internacionales, la que sin embargo se ha ido acortando en la última década.

Esta misma diferencia se registra entre lo ambiental y la responsabilidad social. Lo ambiental implica el cumplimiento de la ley, la verificación del 
respeto a los instrumentos de gestión ambiental. La meta es la ley. La responsabilidad social, en cambio, es la que se desarrolla más allá del simple cumplimiento de la ley. El simple cumplimiento de la ley no convierte a una empresa o a una entidad en socialmente responsable.

En las directrices de la OCDE para empresas multinacionales ${ }^{5}$ se señala que:

[...] las expectativas de los ciudadanos de una mejora del desempeño ambiental se han inculcado a las empresas por medio de una legislación y una reglamentación ambientales más estrictas, y por medio del activismo de la sociedad civil. Muchas empresas tienen ahora un enfoque más proactivo, incluyendo objetivos de mejora continua de sus resultados medioambientales dentro de sus declaraciones de desarrollo sostenible.

Es decir, no basta cumplir con la ley ni con las regulaciones estrictas para actuar bajo principios de responsabilidad social. Se requiere de una acción proactiva orientada hacia la mejora continua de los resultados medio ambientales, y ello solo se logra cuando se incorporan los elementos que resultan centrales: visión integral de futuro, nueva forma de organización y proyección interna y externa.

Sobre ello, Caravedo ${ }^{6}$ señala:

[...] la acción de responsabilidad social implica, pues, varias cosas. En primer lugar, que las empresas desarrollen una visión integral de futuro en la que no sólo está incorporada la comunidad sobre la cual se asientan, sino, también, su país o su sociedad, en un sentido más extenso. En segundo lugar que emerja, una nueva forma de organización, que promueva liderazgos internos, los mismos que contribuyen a reforzar la misión que se traza la empresa y la descentralización de los niveles de autoridad, mejorándose la productividad empresarial. En tercer lugar, su proyección interna (dentro de la empresa) y externa (hacia su entorno externo) la lleva a movilizar no sólo dinero y equipos; sus aportes se hacen, también en recursos humanos y profesionales, dando tiempo para que los propios trabajadores aporten su conocimiento a las diversas actividades que se desarrollan en la sociedad.

Con esa base, las iniciativas para la mejora ambiental continua forman parte integrante de la estrategia de responsabilidad social.

En conclusión, las estrategias de responsabilidad social adecuadamente articuladas pueden resultar un elemento dinamizador de acciones ambientales que sobrepasan el mero cumplimiento de la ley. La política ambiental se puede nutrir activamente de acciones social y ambiental responsables, articuladas bajo la forma de una estrategia organizada, con

5 OCDE. El medio ambiente y las líneas directrices de la ECDE para empresas multinacionales. Herramientas y enfoques empresariales. OECD, 2006, p. 104.

6 CARAVEDo, Baltazar. Perú: empresas responsables. Lima: SASE - Servicios para el Desarrollo y Perú 2021, una nueva visión, 1998, p. 32. 
metas claras e indicadores. Pero, por su lado, no puede haber responsabilidad social sin el cumplimiento mínimo de la ley ambiental.

Ello nos permite identificar el reto futuro. Como en el ámbito del Derecho laboral, la norma ambiental deberá evolucionar para convertirse en una base y dejar de ser, como lo es en la actualidad, una meta. Para ello tiene que retornar a su esencia, que es la protección del derecho fundamental a un ambiente sano, y contar con mecanismos que la hagan exigible, como mínimo, a cumplir.

\section{LAS HERRAMIENTAS DEL DESARROLLO SOSTENIBLE}

En 1987 la Comisión Mundial del Medio Ambiente y del Desarrollo emitió un informe denominado Nuestro futuro común ${ }^{7}$, que constituye el documento base a partir del cual se desarrolla el concepto que resulta piedra angular en el desarrollo de políticas ambientales y de responsabilidad social: el desarrollo sostenible.

Define al desarrollo sostenible como el que satisface las necesidades de la generación presente sin comprometer la capacidad de las generaciones futuras para satisfacer sus propias necesidades. Señala que el desarrollo sostenible es un proceso de cambio en el cual la explotación de los recursos, la orientación de la evolución tecnológica y la modificación de las instituciones son acordes y acrecientan el potencial actual y futuro para satisfacer las necesidades y las aspiraciones humanas.

En esta lógica, el informe señala un conjunto de estrategias urgentes, dentro de las que se destacan:

- revitalizar el crecimiento;

- cambiar la calidad del crecimiento;

- satisfacer las necesidades esenciales de trabajo, alimentos, energía, agua e higiene;

- asegurar un nivel de población sostenible;

- conservar y acrecentar la base de recursos;

- reorientar la tecnología y controlar los riesgos; y

- tener en cuenta el medio ambiente y la economía en la adopción de decisiones.

En consideración a ello, son condiciones básicas para el desarrollo sostenible un sistema político, democrático, que asegure la participación en la toma de decisiones; un sistema económico capaz de crear excedentes y conocimiento técnico; un sistema social que evite las tensiones provocadas por un desarrollo equivocado; un sistema de producción que cumpla con el imperativo de preservar el medio ambiente; un sistema 
tecnológico capaz de investigar nuevas soluciones; un sistema internacional que promueva modelos duraderos de comercio y finanzas; y un sistema administrativo flexible.

Si bien es cierto que estas condiciones están pensadas en la lógica de Estados, es claro que los mismos principios resultan aplicables en la organización empresarial y en las estrategias de responsabilidad social.

Una empresa o entidad socialmente responsable cuenta con una estructura organizativa que facilita la participación, la innovación, los aportes; se articula con el personal de manera de generar relaciones equitativas, informadas; tiene como meta central la protección ambiental y la prevención de impactos potenciales de su actividad; es proactiva y está en constante búsqueda de nuevas soluciones frente a nuevos retos; y es administrativamente flexible y adaptable a responsabilidades que resultan transversales.

Es en esa lógica que las empresas socialmente responsables organizan reportes de sostenibilidad, en el entendido de que:

[...] uno de los retos más importantes que conlleva el desarrollo sostenible es que exige alternativas innovadoras y nuevas formas de pensar. Si bien los avances en conocimientos y tecnologías contribuyen al desarrollo económico, también tienen el potencial de ayudar a resolver los riesgos y amenazas de la sostenibilidad de nuestras relaciones sociales, del medio ambiente y de las economías. Los nuevos conocimientos y las innovaciones en materia de tecnología, gestión y políticas públicas brindan a las organizaciones la oportunidad de elegir nuevas alternativas sobre el modo en el que sus operaciones, productos, servicios y demás actividades influyen en el planeta, en la población y en las economías ${ }^{8}$.

El reto de la sostenibilidad no es, sin embargo, simple de cumplirse, y a nivel empresarial requiere una serie de elementos que permitan una articulación coherente de acciones orientadas a tal fin. Así, una propuesta de sostenibilidad debe contener una serie de acciones sistémicas donde se integren las variables económica, social y ambiental; con elementos tangibles que incluyan estructura, sistemas, procesos y personal; elementos intangibles, como elemento central, constituidos por conductas, estilos y habilidades; y finalmente debe contar con un propósito determinado, claramente expresado.

La dificultad de una estrategia de sostenibilidad nace, no obstante, de su naturaleza de estrategia de largo plazo, la que se enfrenta a necesidades y demandas de corto plazo, tanto en lo económico, pues depende de la capacidad económica para asumir las acciones y la estrategia trazada; en lo social, donde toca enfrentar las demandas del entorno 
y las condiciones de pobreza; y en lo político, pues la sostenibilidad no suele estar en la agenda política por no otorgar réditos en el corto plazo.

El reto es combinar la responsabilidad social con una estrategia de sostenibilidad, ya que ello permite definir una visión de futuro, de largo plazo, y salir de la simple coyuntura. En esta articulación, lo ambiental y lo social juegan un rol fundamental: cuando se pretende alcanzar resultados positivos, no puede separarse de lo económico.

\section{UNA ACCIÓN VOLUNTARIA}

De Belaunde ${ }^{9}$ señala que «la responsabilidad social empresarial plantea, en el ordenamiento jurídico, la conveniencia de encontrar un adecuado balance entre la necesidad de establecer normas legales que regulen y fomenten de manera específica las prácticas empresariales socialmente responsables, o de crear mecanismos que - si bien tienen componente legal - trasciendan la expedición de normas especiales o la modificación de las existentes».

Es que la responsabilidad social es de naturaleza eminentemente voluntaria, y por ello no requiere de un marco legal que la impulse, aunque ello no significa que no deban dictarse algunas regulaciones especiales que la promocionen, en marcos como el tributario, por citar tan solo un ejemplo.

Este carácter voluntario hace que la responsabilidad social no deba ser entendida como una obligación legal o como un procedimiento administrativo. Como se señaló anteriormente, la responsabilidad social implica la incorporación de acciones coherentes, organizadas de manera sistémica, pero a su vez de conductas, filosofía de acción, actitudes.

Una correcta política de responsabilidad social puede conducir a obtener lo que ha venido en denominarse la «licencia social», que puede ser definida como el grado de aprobación que el entorno social, la comunidad o la población le otorgan a una actividad determinada.

Por su naturaleza, la licencia social no puede ser regulada y no puede formar parte de un procedimiento administrativo. Su consecución no permite obtener un documento de certificación, y su obtención no garantiza un plazo determinado de vigencia.

Ese es el reto de la licencia social, ya que su naturaleza obliga a mantener las condiciones de forma tal que el entorno no prive a la actividad de esta licencia; ello requiere seguimiento, adaptación, diálogo permanente y participación. Los que han tratado de asimilar la licencia social a los elementos de la licencia ambiental fallan en tratar de asimilar una herramienta que responde más a una conducta determinada que a un 
procedimiento administrativo que se deriva fundamentalmente de un instrumento, como es la Evaluación del Impacto Ambiental.

La Evaluación del Impacto Ambiental es un proceso de cuatro etapas: identificación y clasificación; preparación y análisis; calificación y decisión; y control y seguimiento. De este se deriva una licencia o una certificación ambiental. Incorpora herramientas de participación y de consulta, e instrumentos de supervisión y de monitoreo. Estas están a cargo de una autoridad, que en el caso peruano es la autoridad sectorial, y están sujetas a una revisión aleatoria de la autoridad ambiental, es decir, del Ministerio del Ambiente.

Esta lógica no está presente en el caso de la licencia social. No hay autoridad a cargo, no hay procedimiento, no hay certificación, no hay etapas. Es un proceso no-formal, pero que requiere de estrategias muy formales. No es posible obtener licencia social si no se cuenta con una estrategia de responsabilidad social, con una estructura organizativa, con el compromiso de todos los ejecutivos y trabajadores de una empresa o entidad, con acciones coherentes, entre otros elementos.

En ese sentido, iniciativas como las presentadas hace algún tiempo en el Congreso de la República para regular la licencia social no solo no resultan necesarias sino que son, en realidad, poco convenientes. Incluso si alguna de ellas se aprobara, resultaría inútil en el objetivo central de obtener esta licencia.

La licencia social se vincula al concepto de «consentimiento informado previo», que empieza a ser reconocido como un derecho que implica el derecho de las comunidades o de los pobladores del entorno de una actividad a estar informados, el derecho a obtener el consenso sobre su desarrollo, el derecho al reconocimiento de la diversidad de los grupos sociales, entre otros componentes.

De acuerdo con el Environmental Law Institute de los Estados Unidos: «El consentimiento puede proveer a las compañías mineras de una 'licencia social' para operar, permitiéndole a la compañía mejorar sus relaciones con la comunidad y, por lo tanto, reducir el riesgo de incurrir en costos originados por conflictos y demoras» ${ }^{10}$.

La licencia social, el consentimiento informado previo y el carácter voluntario de las acciones son elementos que dinamizan el comportamiento ambiental. No puede existir licencia social sin responsabilidad ambiental. Si entendemos, en la lógica de la sostenibilidad, que las mayores demandas sociales no son únicamente por beneficios, sino a su vez por el mantenimiento de las condiciones ambientales que son el sustento de sus actividades habituales, podremos entender que lo ambiental resulta indesligable de la licencia social. 
En este caso, como ya se mencionó, el componente ambiental está más allá de tan solo el cumplimiento de la ley ambiental. En la obtención de la licencia social entran en juego de manera muy significativa las percepciones sobre el comportamiento de una empresa o de una entidad. Las poblaciones conocen del impacto ambiental de las actividades económicas, temen que puedan generar una afectación de sus formas tradicionales de organización y vida y, por ello, muchas veces rechazan su presencia. El comportamiento ambiental, en consecuencia, debe venir acompañado de información, diálogo y generación de confianza.

\section{ENTENDIENDO EL ENTORNO Y PROMOVIENDO LA PARTICIPACIÓN}

La responsabilidad social posee un componente interno, la empresa y sus trabajadores, y un componente externo, el entorno social y ambiental de la empresa, entidad o actividad; $y$, como hemos venido señalando a lo largo de este artículo, requiere acciones sustentadas en la participación, el diálogo y la búsqueda del consenso.

La participación debe entenderse como un proceso a través del cual los intereses y preocupaciones prioritarias, las necesidades y los valores de los ciudadanos son incorporados dentro de la toma de decisiones ${ }^{11}$.

Para lograr un resultado eficaz de la participación, las empresas o las entidades socialmente responsables deben contar con un plan de consulta y participación que contenga una serie de elementos dentro de los que se deben mencionar ${ }^{12}$ :

a. Planificar el proceso con el fin de identificar los riesgos, el contexto social y ambiental, los actores clave, los requerimientos legales.

b. Someter a prueba el programa. Para ello hay que preguntarse si el programa identifica adecuadamente a los actores relevantes, si informa a estos actores sobre los potenciales impactos del proyecto, si provee oportunidad y tiempo para su consideración y respuesta, si responde a todas las preocupaciones y si comunica adecuadamente estas respuestas.

c. Invertir tiempo y recursos, ya que un programa de consulta y participación requiere inversión que garantice el futuro del proyecto.

d. Involucrar directamente a los operadores. No es suficiente involucrar a los funcionarios, sino especialmente a los que desarrollan su actividad en el campo. 
e. Contratar y entrenar al personal correcto, lo cual implica contar con una persona de enlace que se relacione adecuadamente con el ámbito social.

f. Mantener la responsabilidad en el seguimiento integral del programa y sus resultados.

g. Coordinar todas las actividades de participación y de consulta.

h. Construir diálogo y confianza.

i. Manejar las expectativas.

j. Trabajar con el gobierno.

k. Trabajar con ONG y organizaciones sociales de base.

Todo lo mencionado no es suficiente, si a la vez no se cumplen con cuatro principios de gestión básicos:

- poner a disposición la información de manera oportuna;

- usar esta información para la participación y la consulta;

- proveer información entendible; y

- asegurar el acceso a dicha información.

La participación, entendida en sus tres aproximaciones de acceso a la información, acceso al proceso y acceso a la justicia, es un elemento central en una estrategia de responsabilidad social empresarial, y lo es, a su vez, como elemento central de una política ambiental.

Así, la Declaración de Río ${ }^{13}$ suscrita en el marco de la Cumbre de las Naciones Unidas sobre Medio Ambiente y Desarrollo, en 1992, incorporó el Principio 10, que es uno de los más importantes en gestión ambiental a nivel internacional y nacional. Este principio señala que:

El mejor modo de tratar las cuestiones ambientales es con la participación de todos los ciudadanos interesados, en el nivel que corresponda. En el plano nacional, toda persona deberá tener acceso adecuado a la información sobre el medio ambiente de que dispongan las autoridades públicas, incluida la información sobre los materiales y las actividades que encierran peligro en sus comunidades, así como la oportunidad de participar en los procesos de adopción de decisiones. Los Estados deberán facilitar y fomentar la sensibilización y la participación de la población poniendo la información a disposición de todos. Deberá proporcionarse acceso efectivo a los procedimientos judiciales y administrativos, entre éstos el resarcimiento de daños y los recursos pertinentes.

En el Perú se han registrado avances significativos en materia de acceso a la información, con mecanismos sectoriales que garantizan este derecho al ciudadano, pero lamentablemente ello no ocurre en 
todas las entidades del gobierno nacional y menos aún en los niveles subnacionales. A nivel del acceso al proceso, fundamentalmente de manera práctica se ha generado una cultura de prepublicación de normas ambientales, previa a su promulgación, pero no se ha avanzado significativamente en el tema de consulta previa, en especial a poblaciones indígenas en cumplimiento del Convenio 169 de la Organización Internacional del Trabajo.

En lo que se refiere al acceso a la justicia, si bien es cierto se garantiza su acceso al ciudadano en defensa del derecho a un ambiente sano, aun en los casos en que se haya visto directamente afectado (legitimidad para accionar) no existen acciones judiciales ad hoc para la defensa de estos derechos ni tribunales especiales para tal fin. A nivel de la Fiscalía de la Nación, se han creado recientemente las fiscalías ambientales, lo que constituye un paso importante en el fortalecimiento de las reglas de participación.

A nivel privado, en cambio, no existe aún una cultura de transparencia de la información, sustentada en principios de confidencialidad, secreto comercial o industrial y otros equivalentes. Ello genera desconfianza en la población y dificulta las estrategias de responsabilidad social. Por su lado, son pocos los casos en que se promueve la participación en la forma de acceso al proceso, y este se registra fundamentalmente en cumplimiento de la exigencia legal, bajo la forma de audiencias públicas requeridas en ciertos casos de desarrollo de actividades económicas.

Otro tema en el que, como se ha mencionado, no se ha desarrollado el marco legislativo es el de consulta a poblaciones indígenas afectadas por el desarrollo de actividades económicas en sus tierras. Dicha ausencia de consulta ha generado recientes conflictos, como el denominado «conflicto de Bagua», originado en la oposición a la vigencia de algunos decretos legislativos, en especial el 1064 sobre tierras y el 1090 sobre bosques, los que finalmente han sido derogados.

Esta limitación en el desarrollo de la consulta tiene que ver fundamentalmente con la dificultad de responder con precisión a preguntas que requerirían ser materializadas en la norma. ¿Qué se consulta?, en el entendido de que no solo es aplicable a leyes, sino a su vez a normas de menor jerarquía y, de manera concreta, a actividades económicas. ¿A quién se consulta?, lo que implica precisar lo que constituiría una población «afectada» por la actividad. ¿Quién consulta?, lo cual pasa por definir si se debe crear una entidad con responsabilidad en ello o si se debe consultar cada entidad a cargo de un proyecto normativo o empresas a cargo de una actividad. iEn qué momento se consulta?, lo que lleva a la interrogante de si se consulta sobre un proyecto ya materializado como tal o durante una etapa previa a ello. Y finalmente la pregunta central es icon qué consecuencias se consulta?, lo cual lleva a la vieja discusión respecto de si la consulta debe o no tener efectos vinculantes. 
La inexistencia de estas normas genera un obstáculo significativo para las estrategias de responsabilidad social y para el avance y la consolidación de políticas y de regulaciones ambientales. En ese sentido, constituye uno de los retos más importantes para el futuro cercano.

\section{LA RESPONSABILIDAD SOCIALCOMOPRÁCTICA INCLUSIVA}

La responsabilidad social empresarial, reconocida como una práctica que se desarrolla de manera voluntaria, sistémica, más allá del cumplimiento de la ley, no es una medida orientada a obtener tan solo beneficio en el ámbito empresarial. Es más bien una acción que busca integrar el entendimiento del entorno y la consideración a sus problemas mayores, en las acciones de responsabilidad de una empresa.

En esa lógica, la responsabilidad social demanda acciones no solo desde el ámbito empresarial, sino a su vez desde el accionar ciudadano. Los elementos de la responsabilidad social pueden ser adaptados al accionar ciudadano y su relación con el entorno, y generarán, como consecuencia, una política de integración e inclusión que el Perú demanda.

En ese orden de ideas, los términos «responsabilidad social empresarial» o «responsabilidad social corporativa» terminan siendo limitativos, no solo por el hecho de parecer entender lo social como separado o aislado de lo ambiental, sino a su vez porque parecen orientarse tan solo hacia un sector de la economía nacional: el privado, organizado bajo la forma empresarial.

Baltazar Caravedo, a través de su iniciativa «Responsabilidad social: todos» ${ }^{14}$, constituye un buen paso para avanzar en la difusión de la responsabilidad social en un enfoque ciudadano. Como parte de sus reflexiones, Caravedo señala que un primer eje de las preocupaciones radica en convertir la responsabilidad social en parte de la conciencia y de la política de las organizaciones. Agrega, como segunda reflexión, la necesidad de articular alianzas para el desarrollo. Otro eje central es el educativo y, finalmente, se refiere a la comunicación como elemento de transmitir comportamientos responsables.

\section{CONCLUSIONES}

- De manera conceptual, los términos «política» y «gestión ambiental» $\mathrm{y}$ «responsabilidad social empresarial» reúnen principios y elementos complementarios, que se apoyan y retroalimentan entre sí, que hacen que no pueda existir responsabilidad social empresarial sin responsabilidad ambiental y que, a su vez,

14 CARAVEDo, Baltazar (coordinador general). «Responsabilidad social: todos. Hacia un encuentro de todos los peruanos". Lima. 
lo ambiental se fortalezca con la lógica de la responsabilidad: ir más allá del simple cumplimiento de la ley ambiental.

- Las regulaciones ambientales se han enfocado fundamentalmente en el cumplimiento de instrumentos y de estándares de gestión ambiental, a partir de lo cual se han distanciado de su elemento central: la protección del derecho fundamental a un ambiente sano. Ello genera dificultades en la integración de lo ambiental a la corriente de protección de derechos humanos con políticas y estrategias de responsabilidad social. El cumplimiento de instrumentos de gestión ambiental no es el objetivo central de la responsabilidad social. Es el entorno y la persona que, de manera individual o colectiva, forman parte de ese entorno empresarial.

- La responsabilidad social se desarrolla con visión de largo plazo cuando incorpora los elementos del desarrollo sostenible. Ello enfrenta estas estrategias a una serie de obstáculos y dificultades, originadas en las necesidades y demandas de corto plazo, pero al superarlas se construye una visión de futuro que fortalece la responsabilidad.

- La responsabilidad social, la licencia social, es de carácter eminentemente voluntario. Como tal, no requiere de norma o de exigencia legal alguna para su cumplimiento, y el entendimiento con el entorno no deriva de un procedimiento administrativo. Ello obliga a atender no solo los elementos materiales sino a mantener conductas, filosofías y acciones que conduzcan a mantener el consenso con el entorno. La licencia social no deriva de una certificación, de la presencia de una autoridad pública, sino únicamente de la capacidad de la entidad por generar el consenso a través del diálogo y de la participación.

- La participación ciudadana se comprende en tres elementos (acceso a la información, al proceso y a la justicia) centrales en una estrategia de responsabilidad social y, a su vez, en el diseño de marcos de política ambiental. A ello debe sumarse la consulta, previa e informada, por lo que el reto es resolver los elementos que la dificultan, en especial la definición de las consecuencias que la consulta tendría en el proceso de toma de decisiones.

- La responsabilidad social empresarial debe salir del límite que le impone su propio nombre, ya que es más que tan solo el elemento social, y debe corresponder al accionar de organizaciones no lucrativas y del propio ciudadano. Ese es el mayor reto a alcanzar.

RESPONSABILIDAD SOCIAL Y POLÍTICA AMBIENTAL: COMPLEMENTARIEDADES Y RETOS 